\title{
Randomized comparative trial of cervical block protocols for pain management during hysteroscopic removal of polyps and myomas
}

This article was published in the following Dove Press journal:

International Journal of Women's Health

13 October 2015

Number of times this article has been viewed

\author{
Andrea S Lukes' \\ Kelly H Roy ${ }^{2}$ \\ James B Presthus ${ }^{3}$ \\ Michael P Diamond ${ }^{4}$ \\ Jay M Berman ${ }^{4}$ \\ Kenneth A Konsker \\ 'Carolina Women's Research and \\ Wellness Center, Durham, NC, USA; \\ ${ }^{2}$ Phoenix Gynecology Consultants, \\ Phoenix, AZ, USA; ${ }^{3}$ Minnesota \\ Gynecology and Surgery, Edina, MN, \\ USA; ${ }^{4}$ Wayne State University, Detroit, \\ MI, USA; ${ }^{5}$ Florida Woman Care, Boca \\ Raton, FL, USA
}

Purpose: To evaluate the efficacy of two cervical block protocols for pain management during hysteroscopic removal of intrauterine polyps and myomas using the MyoSure ${ }^{\circledR}$ device.

Patients and methods: This was a randomized, comparative treatment trial conducted by five private Obstetrics and Gynecology practices in the USA. Forty premenopausal women aged 18 years and older were randomized to receive either a combination para/intracervical block protocol of $37 \mathrm{cc}$ local anesthetic administered at six injections sites in association with the application of topic $1 \%$ lidocaine gel, or an intracervical block protocol of $22 \mathrm{cc}$ local anesthetic administered at three injections sites without topical anesthetic, for pain management during hysteroscopic removal of intrauterine polyps and/or a single type 0 or type 1 submucosal myoma $\leq 3 \mathrm{~cm}$. The main outcomes were a composite measure of procedure-related pain and pain during the postoperative recovery period, assessed by the Wong-Baker Faces Rating Scale $(0=$ no pain to $10=$ maximum pain $)$. The lesion characteristics, procedure time, and adverse events were summarized.

Results: A total of 17 polyps and eight myomas were removed in the para/intracervical block group, with diameters of $1.3 \pm 0.5 \mathrm{~cm}$ and $1.8 \pm 0.8 \mathrm{~cm}$, respectively. In the intracervical block group, 25 polyps with a mean diameter of $1.2 \pm 0.7 \mathrm{~cm}$ and 7 myomas with a mean diameter of $1.9 \pm 0.9 \mathrm{~cm}$ were removed. The mean tissue resection time was $1.2 \pm 2.0$ minutes and $1.2 \pm 1.4$ minutes for the para/intracervical and intracervical block groups, respectively. The mean composite procedure-related pain score was low for both cervical block protocols, $1.3 \pm 1.4$ in the para/intracervical block group vs $2.1 \pm 1.5$ in the intracervical block group. During the postoperative recovery period, the mean pain scores were $0.3 \pm 0.7 \mathrm{vs} 1.2 \pm 1.7$ for the para/intracervical and intracervical block groups, respectively. There were no serious adverse events.

Conclusion: The MyoSure procedure for removal of polyps and myomas was well tolerated, with low pain scores reported for both the para/intracervical and intracervical block protocols.

Keywords: MyoSure, hysteroscopic morcellation, polypectomy, myomectomy

\section{Introduction}

Hysteroscopic loop resection has been a mainstay of treatment for the removal of intrauterine pathology, including polyps and myomas. Traditionally, this procedure has been performed in the operating room, with the patient under general anesthesia, primarily due to the use of electrocautery, nonionic distension media, and the need for cervical dilation. ${ }^{1,2}$ While effective, this technique has limitations, including distension media issues, the need for frequent removal and reinsertion of the hysteroscope to remove resected tissue, and the risk of uterine perforation. Loop resection must also be performed with intravenous (IV) sedation or general anesthesia. ${ }^{1,2}$
Correspondence: Andrea S Lukes Carolina Women's Research and Wellness Center, 249 E NC Highway 54, Suite 330, Durham, NC 27713, USA

$\mathrm{Tel}+\mathrm{I} 9192519223$

$\mathrm{Fax}+19192519343$

Email andrealukes@cwrwc.com 
A newer technique, hysteroscopic morcellation, has been shown to be safe and effective for removing intrauterine polyps and myomas. ${ }^{1-3}$ This technique offers several advantages over loop resection, including decreased procedure time, decreased number of instrument removals and reinsertions, a faster learning curve for physicians, and a more rapid recovery. ${ }^{1-4}$ However, when first introduced, the size of hysteroscopic morcellators also required that the procedure be performed in a surgical setting with the use of IV sedation or general anesthesia. Conversely, other hysteroscopic procedures can be performed with local anesthesia, and several studies have shown that paracervical blocks are associated with effective pain management and high rates of patient satisfaction during these procedures. ${ }^{5-8}$

Recent improvements in technology, including smaller sized instruments, indicate that hysteroscopic morcellation can be performed using local anesthesia for pain management, in a similar manner to other hysteroscopic procedures. However, little is known about the use of different cervical block protocols without IV sedation for pain management during hysteroscopic morcellation. Therefore, the goal of this study was to compare the efficacy of two cervical block protocols for pain management during hysteroscopic removal of intrauterine polyps and myomas.

\section{Materials and methods}

This randomized comparative trial ${ }^{9}$ examined the efficacy of two cervical block protocols for pain management during the hysteroscopic removal of intrauterine polyps and myomas. The study was conducted at five sites in the USA, with five investigators (one per site), and included a total of $40 \mathrm{sub}-$ jects. Three investigators performed a total of 20 procedures in their respective offices and two investigators performed a total of 20 procedures at ambulatory surgical centers. The same protocol was followed at all sites.

Premenopausal women aged 18 years or older with intrauterine polyps and/or a single type 0 or type 1 submucosal myoma $\leq 3 \mathrm{~cm}$ were eligible to participate. An indication for polypectomy or myomectomy for benign pathology was determined by transvaginal ultrasound or saline-infused sonogram within 60 days of the procedure. A negative pregnancy test within 48 hours of the procedure and willingness to use reliable birth control for the next 30 days were required. The exclusion criteria included having a type 2 myoma; known or suspected endometrial or other cancers; contraindication or allergy to local anesthetic or oral medications specified in the study protocol; prior uterine artery embolization or any other uterine artery occlusion procedure; an intrauterine device; active pelvic infection; known or suspected hemostatic disorder; use of anticoagulant or antiplatelet medications; a history of chronic narcotics use; or other serious medical conditions.

The subjects were recruited between December 2009 and July 2010 from the investigators' clinical practices and by radio advertisement. Enrollment was offered to all patients meeting the eligibility criteria, and the first 40 patients who agreed to participate were enrolled. The protocol was approved by the Quorum Institutional Review Board (Seattle, WA, USA) and the Wayne State University Institutional Review Board (Detroit, MI, US, USA). All subjects provided written, informed consent and received compensation for their participation.

The subjects were randomized on the day of treatment to either a combination para/intracervical block group or an intracervical block group in a 1:1 ratio, using a computer-generated randomization scheme. The specific randomization assignment was provided to sites using sealed, sequentially numbered opaque envelopes, ${ }^{9}$ which were opened in succession on the day of the treatment procedure. The subjects were blinded to the group assignment because they were not told how many injections either group would be receiving.

\section{Anesthetic protocol}

All subjects received the same preoperative analgesic/anesthetic treatment prior to the administration of the cervical block. The subjects were asked to take $800 \mathrm{mg}$ of ibuprofen the night before the procedure. One hour prior to the procedure, the subjects received $10 \mathrm{mg}$ of diazepam and $10 \mathrm{mg}$ of hydrocodone/acetaminophen, followed by an intramuscular injection of $30 \mathrm{mg}$ keterolac and $0.4 \%$ atropine. Both cervical blocks were a 1:1 mixture of $1 \%$ lidocaine and $0.25 \%$ bupivicaine.

\section{Para/intracervical block protocol}

The para/intracervical block group received a total of $37 \mathrm{cc}$ of anesthetic, administered at six injections sites: $2 \mathrm{cc}$ superficially at 12:00; $10 \mathrm{cc}$ at 3:00; and $10 \mathrm{cc}$ at 9:00, in the cervical vaginal junction, superficially through the mucosa (approximately $0.5 \mathrm{~cm}$ deep); $5 \mathrm{cc}$ at 4:00; $5 \mathrm{cc}$ at 8:00, approximately 1 to $2 \mathrm{~cm}$ deep, halfway between the canal and the lateral edge of the cervix; and 5 cc submucosally at 6:00, between the uterosacral ligaments, approximately $0.5 \mathrm{~cm}$ below the cervical vaginal junction (Figure 1). Topical $1 \%$ lidocaine gel was applied to the cervix, with a set time 


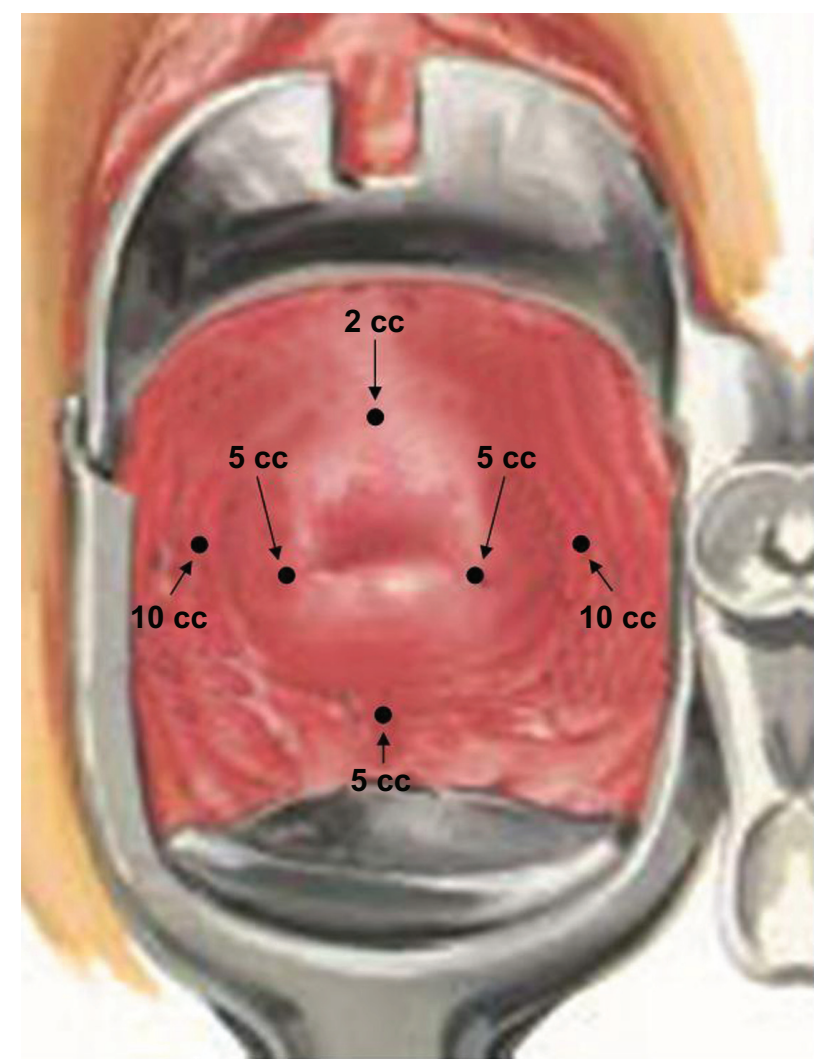

Figure I Para/intracervical block injection sites.

Notes: The para/intracervical block group received a total of $37 \mathrm{cc}$ of anesthetic administered at six injections sites: $2 \mathrm{cc}$ superficially at 12:00; $10 \mathrm{cc}$ at 3:00; and $10 \mathrm{cc}$ at 9:00, in the cervical vaginal junction, superficially through mucosa, approximately $0.5 \mathrm{~cm}$ deep; $5 \mathrm{cc}$ at 4:00; and $5 \mathrm{cc}$ at 8:00, approximately 1 to $2 \mathrm{~cm}$ deep, halfway between the canal and the lateral edge of the cervix; and $5 \mathrm{cc}$ submucosally at 6:00, between the uterosacral ligaments, approximately $0.5 \mathrm{~cm}$ below the cervical vaginal junction. Topical $1 \%$ lidocaine gel was applied to the cervix, with a set time of 2 to 3 minutes prior to the injection of anesthetic, for the para/intracervical block group only. Figure provided courtesy of Mark Glasser, MD.

of 2 to 3 minutes prior to the injection of anesthetic, for the para/intracervical block group only.

\section{Intracervical block protocol}

The intracervical block group received a total of $22 \mathrm{cc}$ of anesthetic administered at three injections sites: 2 cc superficially at 12:00; $10 \mathrm{cc}$ at 4:00; and $10 \mathrm{cc}$ at 8:00, approximately 1 to $2 \mathrm{~cm}$ deep (Figure 2).

\section{The MyoSure procedure}

All procedures were performed using the MyoSure ${ }^{\circledR}$ Tissue Removal System (Hologic, Inc, Bedford, MA, USA). The MyoSure system is a suction-based, mechanical energy system that removes intrauterine tissue, using a $2.5 \mathrm{~mm}$ cutting blade (which oscillates and reciprocates at speeds up to $6,000 \mathrm{rpm}$ ) within a $3 \mathrm{~mm}$ outer tube with a side-facing window (Figure 3). ${ }^{10}$ After a 10 -minute induction period for the anesthetic, the cervix was dilated using a 6 French Hegar

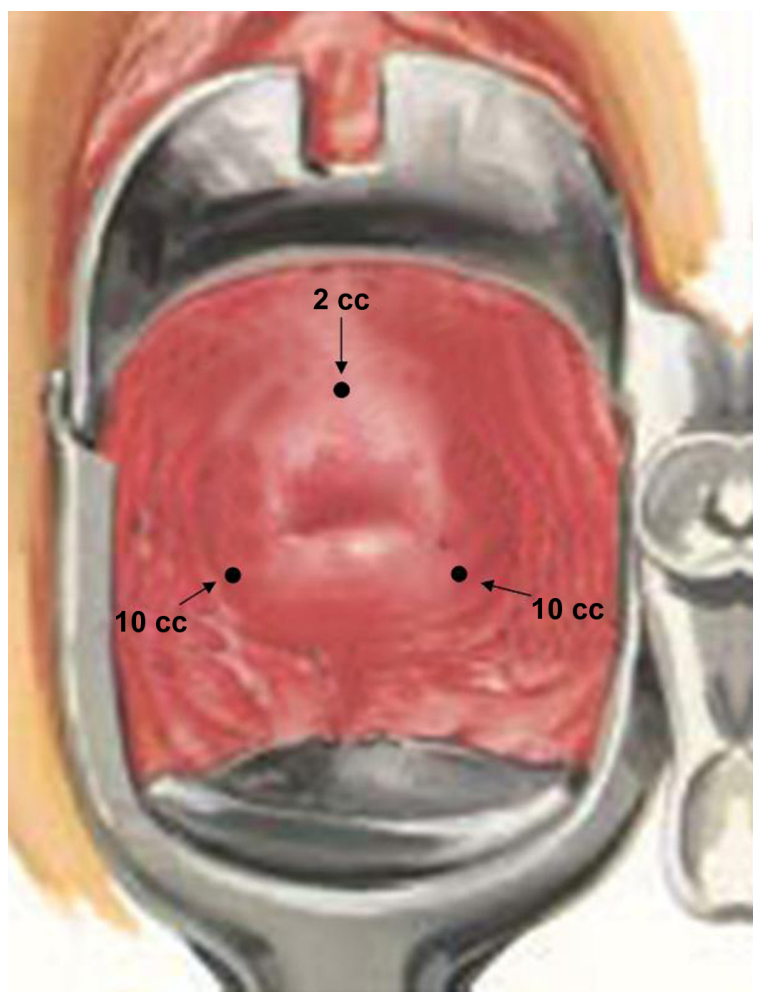

Figure 2 Intracervical block injection sites.

Notes: The intracervical block group received a total of $22 \mathrm{cc}$ of I\% lidocaine and $0.25 \%$ bupivacaine, administered at three injections sites: 2 cc superficially at 12:00; $10 \mathrm{cc}$ at 4:00; and $10 \mathrm{cc}$ at 8:00, approximately $\mathrm{I}$ to $2 \mathrm{~cm}$ deep. Figure provided courtesy of Mark Glasser, MD.

dilator, and the MyoSure device was introduced into the uterus through a $6.25 \mathrm{~mm}$ offset lens, $0^{\circ}$, custom-designed hysteroscope. Sterile saline solution was used for distension. The target lesions were resected under direct visualization and suctioned from the cavity into a collection canister.

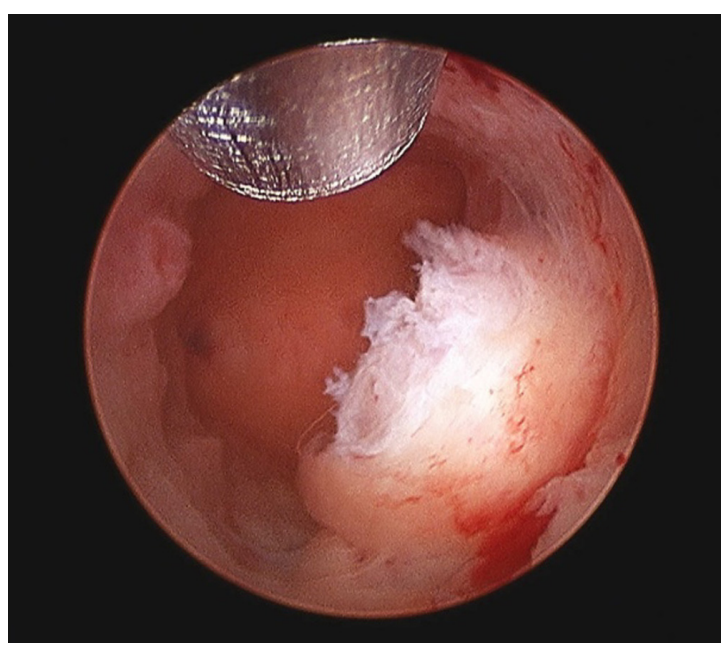

Figure 3 Hysteroscopic image of the MyoSure ${ }^{\circledR}$ tissue removal device in the uterine cavity.

Note: Resection of myomatous tissue with the MyoSure device, midway through the procedure. 
The investigators or study personnel communicated the steps of the procedure to the subjects as they occurred. The type of lesion, size, location, and the estimated percent volume removed were assessed by the investigators. All the resected tissue was sent for pathological evaluation. Rescue anesthesia was available in the event that the cervical block proved inadequate.

\section{Outcome measures}

The main outcome was a composite score for procedure-related pain, which incorporated individual pain scores during: 1) the cervical block injection; 2) cervical dilation; 3) uterine distention; and 4) the tissue resection. The procedure-related pain for each stage of the procedure was assessed immediately after the entire procedure was complete, prior to transfer to the recovery room, using the Wong-Baker Faces Rating Scale. ${ }^{11}$ This scale provides a score ranging from 0 , indicating "no pain," to 10 , indicating "maximum pain." Pain during the postoperative recovery period prior to return home was a secondary outcome. The exploratory outcomes included the physician and subject satisfaction with the cervical block, ranging from "very dissatisfied" to "very satisfied".

The adverse events that occurred during the procedure or postoperative recovery period were recorded. Follow up was done by telephone at 48 hours, and at 7 and 30 days postprocedure to determine the resolution of any operative adverse events and whether adverse events occurred after the return home.

\section{Statistical analysis}

A total sample size of 40 subjects for the pooled sites was estimated to provide $86 \%$ power to detect a mean difference of 1.0 in procedure-related pain. Because no previous studies have examined pain with different types of cervical blocks during hysteroscopic morcellation, the sample size was estimated based on reported pain scores when using local anesthetic for cervical dilation and uterine dilation. ${ }^{12}$ This study was not powered to detect a difference in serious adverse events, due to the low incidence of these events associated with hysteroscopic morcellation. ${ }^{3,13}$ All statistical analyses were performed using a modified intent-to-treat model. This included subjects who met the inclusion and exclusion criteria and did not have a major protocol deviation. The primary outcome of procedure-related pain and the secondary outcome of postprocedure recovery pain were analyzed using a one-way analysis of variance (ANOVA), with the factors of cervical block group and investigator. All of the statistical analysis was performed using SAS ${ }^{\circledR}$ software version 9.1
(SAS Institute Inc, Carey, NC, USA). $P$-values $<0.05$ were considered significant.

\section{Results}

Of the 40 subjects who were enrolled in the study, 19 were randomized to the combination para/intracervical block group and 21 were randomized to the intracervical block group. All randomized subjects underwent the procedure; however, two subjects failed to meet the inclusion and exclusion criteria, in one case, this was because of narcotics use and in the other, because of a Type 2 myoma ( $<50 \%$ in the uterine cavity); and one subject had a major protocol deviation - in this case, the investigator converted to a loop resection procedure, due to a vascularized type I myoma located within the uterine fundus. As a result, the final analysis included 17 subjects in the para/intracervical block group and 20 subjects in the intracervical block group.

There were no significant differences in the baseline demographic characteristics, parity, or the gynecological history between the groups (Table 1). There were also no significant differences in the combined number of polyps and myomas (total lesions), the mean size of the polyps and myomas, or in the intrauterine location of lesions between the groups (Table 2). The majority of subjects in both groups had one lesion, $70.5 \%$ in the para/intracervical group and $55.0 \%$ in the intracervical group (Table 2). It was intraoperatively

Table I Subject demographic characteristics and gynecological history

\begin{tabular}{|c|c|c|c|}
\hline & $\begin{array}{l}\text { Paral } \\
\text { Intracervical } \\
(n=I 7)\end{array}$ & $\begin{array}{l}\text { Intracervical } \\
(n=20)\end{array}$ & $P$-value \\
\hline Age, years, mean \pm SD & $44.2 \pm 7.7$ & $41.8 \pm 7.5$ & $0.26^{\mathrm{a}}$ \\
\hline Race, n (\%) & & & $0.12^{\mathrm{b}}$ \\
\hline Caucasian & 7 (4I.2\%) & $10(50.0 \%)$ & \\
\hline African American & $6(35.3 \%)$ & $10(50.0 \%)$ & \\
\hline Hispanic/Latino & $3(17.6 \%)$ & $0(0.0 \%)$ & \\
\hline Asian & I (5.9\%) & $0(0.0 \%)$ & \\
\hline Parity, n (\%) & & & $0.18^{b}$ \\
\hline Nulliparous & $6(35.3 \%)$ & $10(50.0 \%)$ & \\
\hline Parous & II (64.7\%) & $10(50.0 \%)$ & \\
\hline Prior treatment(s) & & & $0.53^{b}$ \\
\hline \multicolumn{4}{|l|}{ for myomas, n (\%) } \\
\hline Yes & $3(17.6 \%)$ & $2(10.0 \%)$ & \\
\hline No & $14(82.4 \%)$ & $18(90.0 \%)$ & \\
\hline $\begin{array}{l}\text { History of abnormal } \\
\text { uterine bleeding, } \mathrm{n}(\%)\end{array}$ & & & $0.24^{b}$ \\
\hline Yes & $13(76.5 \%)$ & I8 (90.0\%) & \\
\hline No & $4(23.5 \%)$ & $2(10.0 \%)$ & \\
\hline
\end{tabular}

Notes: ${ }^{a}$ ANOVA with the factors of cervical block and investigator; ${ }^{\text {b Cochran- }}$ Mantel-Haenszel test, stratified by investigator.

Abbreviations: ANOVA, analysis of variance; SD, standard deviation. 
Table 2 Summary of lesions, by hysteroscopic evaluation

\begin{tabular}{|c|c|c|c|}
\hline & $\begin{array}{l}\text { Paral } \\
\text { Intracervical } \\
(n=\mid 7)\end{array}$ & $\begin{array}{l}\text { Intracervical } \\
(n=20)\end{array}$ & $P$-value \\
\hline Total lesions, $n$ & 26 & 32 & $0.9 I^{\mathrm{a}}$ \\
\hline Polyps, $\mathrm{n}$ & 17 & 25 & \\
\hline Diameter, cm & $1.3 \pm 0.5$ & $1.2 \pm 0.7$ & \\
\hline \% Removed & $95.5 \pm 15.1$ & $98.1 \pm 9.6$ & \\
\hline Myomas, n & 8 & 7 & \\
\hline Diameter, cm & $1.8 \pm 0.8$ & $1.9 \pm 0.9$ & \\
\hline$\%$ Removed & $90.6 \pm 22.8$ & $95.0 \pm 16.8$ & \\
\hline $\begin{array}{l}\text { Number of lesions by } \\
\text { number of subjects, } n \text { (\%) }\end{array}$ & & & $0.99^{\mathrm{a}}$ \\
\hline I & $12(70.5 \%)$ & II (55.0\%) & \\
\hline 2 & $2(1 \mathrm{I} .8 \%)$ & $6(30.0 \%)$ & \\
\hline 3 & $2(11.8 \%)$ & $3(15.0 \%)$ & \\
\hline $4+$ & I (5.9\%) & - & \\
\hline \multicolumn{4}{|c|}{ Total lesions by location, $\mathrm{n}(\%)^{\mathrm{b}}$} \\
\hline Anterior & 7 (28.0\%) & $9(28.1 \%)$ & 0.88 \\
\hline Posterior & $6(24.0 \%)$ & $10(31.2 \%)$ & 0.58 \\
\hline Fundal & $6(24.0 \%)$ & $6(18.8 \%)$ & 0.48 \\
\hline Other & $6(24.0 \%)$ & $7(21.9 \%)$ & 1.00 \\
\hline
\end{tabular}

Notes: ${ }^{a}$ Cochran-Mantel-Haenszel test for row mean score, stratified by investigator; 'subjects may have $\geq 1$ lesion. The location of one lesion was not recorded for one subject in the para/intracervical block group.

estimated that $95.5 \% \pm 15.1 \%$ and $98.1 \% \pm 9.6 \%$ of polyp volume was removed in the para/intracervical and intracervical block groups, respectively. The mean myoma volume removed was estimated to be $90.6 \% \pm 22.8 \%$ in the para/ intracervical block group and $95.0 \% \pm 16.8 \%$ in the intracervical block group. The mean procedure time from the injection of the cervical block to the completion of tissue removal was $29.2 \pm 10.0$ minutes for the para/intracervical block group and 28.2 \pm 7.5 minutes for the intracervical block group. The tissue resection time was $1.2 \pm 2.0$ minutes and was $1.2 \pm 1.4$ minutes for the para/intracervical and intracervical block groups, respectively.

The composite procedure-related pain score was low in both groups. However, there was a statistically significant difference in pain score between the para/intracervical block group and the intracervical block group, $1.3 \pm 1.4$ vs $2.1 \pm 1.5$, respectively $(P<0.05)$ (Table 3$)$. The pain during the tissue resection phase of the procedure and the postprocedure recovery pain prior to return home were also lower in the para/ intracervical block group compared with the intracervical block group (Table 3). Finally, there was a trend towards lower pain during uterine distension in the para/intracervical block group as compared with the intracervical block group.

All the investigators (100\%) reported being "very satisfied" with the para/intracervical block performance, and $95.0 \%$ reported being "very satisfied" with the
Table 3 Procedure-related and postprocedure recovery pain scores

\begin{tabular}{|c|c|c|c|}
\hline Pain score $^{a}$ & $\begin{array}{l}\text { Paral } \\
\text { Intracervical } \\
(n=\mid 7)\end{array}$ & $\begin{array}{l}\text { Intracervical } \\
(n=20)\end{array}$ & $P$-value \\
\hline $\begin{array}{l}\text { Composite procedure- } \\
\text { related }\end{array}$ & $1.3 \pm 1.4$ & $2.1 \pm 1.5$ & 0.05 \\
\hline \multicolumn{4}{|l|}{ Procedure phase } \\
\hline Cervical block injection & $2.1 \pm 2.7$ & $2.4 \pm 1.7$ & 0.42 \\
\hline Cervical dilation & $1.6 \pm 2.1$ & $2.6 \pm 2.1$ & 0.09 \\
\hline Uterine distention & $1.0 \pm 1.5$ & $1.8 \pm 1.8$ & 0.06 \\
\hline Tissue resection & $0.7 \pm 1.3$ & $1.7 \pm 2.0$ & 0.05 \\
\hline Postprocedure recovery & $0.3 \pm 0.7$ & $1.2 \pm 1.7$ & 0.02 \\
\hline
\end{tabular}

Note: ${ }^{\text {aAll }}$ values are mean \pm SD.

Abbreviation: SD, standard deviation.

intracervical block. In one case, an investigator reported being "somewhat dissatisfied" with the intracervical block. The subjects in the para/intracervical and intracervical block groups were "very satisfied" with pain management in $88.2 \%$ and $85.9 \%$ of cases, respectively. The remainder of subjects in both groups were "somewhat satisfied" with the pain management during the procedure.

No serious adverse events occurred. Mild adverse events were reported, including endometritis (three events), fungal infection (one event), and dysmenorrhea (one event). In one case, a previously placed nickel titanium tubal occlusion implant (for sterilization) was removed when the MyoSure device was removed. All adverse events resolved completely without sequelae. No signs of local anesthetic toxicity, fluid intravasation, significant bleeding, or uterine perforations occurred during the study. No subjects required rescue anesthesia during the procedure.

\section{Discussion}

This study demonstrates that the procedure-related pain during hysteroscopic morcellation of intrauterine polyps and myomas was mild when either a combination para/ intracervical or intracervical block was used. Pain during the recovery period was also minimal. Comparative analysis indicated that the para/intracervical block protocol was associated with lower pain compared with an intracervical block However, it is not known whether this difference is clinically significant, nor is it known which particular difference(s) between the pain management protocols contributed to this difference. These results also showed that the majority of physicians and subjects were very satisfied with pain management with either cervical block protocol.

Until recently, the hysteroscopic removal of polyps and myomas has been done by loop resection and required the 
use of IV sedation or general anesthesia. To our knowledge, this is the first study examining the effect of different cervical blocks on pain management during the hysteroscopic morcellation of intrauterine polyps and myomas. Al-Sunaidi and Tulandi compared the effects of a combination para/ intracervical block with a lower-dose intracervical block for pain management during hysteroscopy, but surgery was not performed in that study. ${ }^{14}$ Consistent with our results, the investigators reported that both the para/intracervical and the intracervical blocks were well tolerated and associated with mild pain during the procedure. Also, as noted in our study, the combined para/intracervical block was associated with less pain during the procedure and during the recovery period compared with the intracervical block.

A number of studies compared the effects of a paracervical block only (not combined with intracervical block) with an intracervical block for pain during various gynecologic procedures. ${ }^{12}$ However, it is difficult to compare the results of these studies to ours, given the significant protocol differences.

While both groups reported only mild pain during the procedure and recovery, the lower pain seen in the para/ intracervical block group compared with the intracervical block group may be attributed to several factors. Only the para/intracervical group received a topical lidocaine gel applied to the cervix before injection of the local anesthetic, which may have contributed to pain management. The para/ intracervical block group also received a larger dose of anesthetic, $37 \mathrm{cc}$ vs $22 \mathrm{cc}$, and a greater number of injections, six vs three, compared with the intracervical block group. This may have resulted in greater tissue area dispersion and different effects on innervation.

No significant adverse events occurred during this study. However, the removal of a tubal occlusion implant for sterilization in one subject resulted in a revision to the MyoSure instructions for use. The instructions for use currently indicate that users should exercise extreme caution when resecting tissue in patients who have implants that extend into the uterine cavity and that users do not use the MyoSure tissue removal device to resect tissue that is adjacent to an implant. ${ }^{10}$

This study had some limitations. The sample size was small, and larger studies are needed to compare different cervical block protocols and to identify best practices for pain management during the hysteroscopic removal of intrauterine pathology. Additionally, the Wong-Baker scale can force clustering around predefined values, and this potentially contributed to the relatively small difference in pain scores reported in this study. The results may have been subject to recall bias because the procedure-related pain was recorded during the recovery period. However, steps were taken to decrease any potential timing effects, including the clear communication of the procedure phases as they occurred.

\section{Conclusion}

This study demonstrated that pain during hysteroscopic morcellation of intrauterine polyps and type 0 or 1 myomas can be successfully managed with a local anesthetic. Two different pain management protocols, a combination para/ intracervical block and an intracervical block, were both associated with low pain scores for the hysteroscopic morcellation of uterine pathology.

\section{Acknowledgments}

The authors thank Jennifer Layne, PhD for editorial support.

\section{Disclosure}

This study was sponsored by Hologic, Inc, and all the authors received research support.

The authors report no other conflicts of interest in this work.

\section{References}

1. American Association of Gynecologic Laparoscopists AAGL practice report: AAGL practice report: practice guidelines for the diagnosis and management of submucous leiomyomas. J Minim Invasive Gynecol. 2012;19(2):152-171.

2. American Association of Gynecologic Laparoscopists. AAGL practice report: practice guidelines for the diagnosis and management of endometrial polyps. J Minim Invasive Gynecol. 2012;19(1):3-10.

3. Emanuel MH, Wamsteker K. The intra uterine morcellator: a new hysteroscopic operating technique to remove intrauterine polyps and myomas. J Minim Invasive Gynecol. 2005;12(1):62-66.

4. van Dongen H, Emanuel MH, Wolterbeek R, Trimbos JB, Jansen FW. Hysteroscopic morcellator for removal of intrauterine polyps and myomas: a randomized controlled pilot study among residents in training. J Minim Invasive Gynecol. 2008;15(4):466-471.

5. Arjona JE, Miño M, Cordón J, Povedano B, Pelegrin B, Castelo-Branco C. Satisfaction and tolerance with office hysteroscopic tubal sterilization. Fertil Steril. 2008;90(4):1182-1186.

6. Bettocchi S, Ceci O, Nappi L, et al. Operative office hysteroscopy without anesthesia: analysis of 4863 cases performed with mechanical instruments. J Am Assoc Gynecol Laparosc. 2004;11(1):59-61.

7. Chudnoff S, Einstein M, Levie M. Paracervical block efficacy in office hysteroscopic sterilization: a randomized controlled trial. Obstet Gynecol. 2010;115(1):26-34.

8. Sinha D, Kalathy V, Gupta JK, Clark TJ. The feasibility, success and patient satisfaction associated with outpatient hysteroscopic sterilisation. BJOG. 2007;114(6):676-683.

9. Hologic, Inc. Comparative Sedation Study of the MyoSure Hysteroscopic Tissue Removal System. Available from: http://www.clinicaltrials.gov/ct2/show/NCT00979342?term=myosure\&rank=2. NLM identifier NCT00979342. Accessed November 19, 2013.

10. Interlace Medical. MyoSure ${ }^{\circledR}$ Hysteroscopic Tissue Removal System Instructions for Use. Framingham, MA: Interlace Medical; 2010.

11. Wong DL, Hockenberry-Eaton M, Wilson D, Winkelstein ML, Scwartz P. Wong's Essentials of Pediatric Nursing. 6th ed. St Louis, MO: Mosby; 2001. 
12. Tangsiriwatthana T, Sangkomkamhang US, Lumbiganon P, Laopaiboon M. Paracervical local anaesthesia for cervical dilatation and uterine intervention. Cochrane Database Syst Rev. 2013;9:CD005056.

13. Miller C, Glazerman L, Roy K, Lukes A. Clinical evaluation of a new hysteroscopic morcellator-retrospective case review. $J$ Medicine. 2009;2(3):163-166.
14. Al-Sunaidi M, Tulandi T. A randomized trial comparing local intracervical and combined local and paracervical anesthesia in outpatient hysteroscopy. J Minim Invasive Gynecol. 2007;14(2):153-155.

International Journal of Women's Health

\section{Publish your work in this journal}

The International Journal of Women's Health is an international, peerreviewed open-access journal publishing original research, reports, editorials, reviews and commentaries on all aspects of women's healthcare including gynecology, obstetrics, and breast cancer. The manuscript management system is completely online and includes a very quick and fair peer-review system, which is all easy to use. Visit http://www.dovepress.com/testimonials.php to read real quotes from published authors.

Submit your manuscript here: http://www.dovepress.com/international-journal-of-womens-health-journal 